\title{
Crisis familiares: una oportunidad para transitar de la catástrofe y el caos, al despliegue de capacidades para el cambio*
}

\section{Family Crisis: An Opportunity to Transit from Catastrophe and Chaos into the Deployment of Skills for Change}

\author{
Sandra Milena Valencia** \\ ORCID: 0000-0001-8501-8709 \\ Universidad de Caldas, Manizales, Colombia
}

Recibido: 22 de marzo de 2019 Revisado: 17 de agosto de 2019 Aceptado: 2 de noviembre 2019

\section{Resumen}

El artículo presenta algunos hallazgos derivados de la investigación "Prácticas dialógicas y códigos sociolingüísticos en familias hacia la superación de situaciones de crisis", el cual consistió en comprender, en el diálogo con las familias, el significado que tuvo para su vida y sus relaciones familiares vivir y superar exitosamente situaciones de crisis. Esta investigación siguió una metodología cualitativa y un enfoque hermenéutico, que permitió, a través de entrevistas semiestructuradas a 30 familias urbanas y rurales, residentes en los municipios de Manizales, Neira y Risaralda, acercarse y comprender sus experiencias desde su propia perspectiva. Se concluye que las familias, por sus propios medios y capacidades, resuelven en la mayoría de los casos exitosamente crisis que ocurren en su trayectoria de vida, y que esto genera aprendizajes a nivel personal y familiar.

Palabras clave: cambio; crisis; capacidades; familia

\footnotetext{
Artículo de Investigación. Citar como: Valencia, S. M. (2020). Crisis familiares: una oportunidad para transitar de la catástrofe y el caos, al despliegue de capacidades para el cambio. Diversitas: Perspectivas en Psicología, 16(1), 169-180. Dol: https://doi.org/10.15332/22563067.4115 Este artículo de investigación se basa en los resultados de uno de los objetivos planteados en la investigación "prácticas dialógicas y códigos sociolingüísticos en familias hacia la superación de situaciones de crisis" (inscrita en la Vicerrectoría de Investigaciones y Posgrados de la Universidad de Caldas con código No. 1521414), realizada entre septiembre de 2014 y septiembre de 2016 y, es presentado como requisito para promoción docente, según el acuerdo 15 de 2015 emanado por el consejo superior.

Autor de correspondencia: Sandra Milena Valencia, Docente Departamento de Estudios de Familia. Universidad de Caldas. Manizales, Colombia. Correo electrónico: sandram.valencia@ucaldas.edu.co
} 


\section{Abstract}

This article presents some findings derived from the research titled "Dialogical Practices and Sociolinguistic Codes in Families aimed at overcoming crises", which consisted of understanding, in dialogue with the families, the meaning it had for their lives and their family relations to live through and successfully overcome crises. It was guided by a qualitative methodology and a hermeneutic approach, which enabled, through interviews, to approach and understand such experiences from the perspective of the families themselves. It was evidenced that families solve successfully, in most cases, crises that occur in their life arch, and this by their own means and making use of their skills, which generates learning on the personal and family levels.

Keywords: change; crisis; skills; family.

\section{Introducción}

La familia es un escenario a través del cual es posible construir y promover procesos de cambio y cuya finalidad es el desarrollo óptimo de todos sus integrantes. Aunque ésta es una premisa básica de los procesos de acompañamiento familiar, fue común encontrar tanto en las investigaciones como en los procesos de prácticas, realizados por profesionales y estudiantes de distintas áreas del conocimiento, una tendencia a la utilización de lenguajes del déficit. Dicha revisión de antecedentes se llevó a cabo por parte del equipo de investigación entre el año 2014 y el 2016. Esta postura encontrada poco trasciende la identificación de situaciones y condiciones problémicas que enfrentan cotidianamente los integrantes de las familias. Si bien, en los propósitos de las investigaciones aparecen ejes temáticos como: el "cambio", la "resiliencia”, la "transformación", el "proyecto de vida" y los "recursos", la presentación de los resultados no da cuenta explícitamente de la manera cómo la población sobre la cual se realizan las investigaciones, asume e incorpora estos procesos.

Así mismo, en estas investigaciones tampoco se da cuenta de la utilización de lenguajes creativos y generativos en los que se hicieran visibles las capacidades (recursos y estrategias) activadas e implementadas por las familias en su trayectoria de vida, para afrontar las situaciones y condiciones cotidianas que obstaculizan su desarrollo. Estas herramientas son propias del enfoque apreciativo y útiles en el reconocimiento de las familias como agentes de cambio. En este mismo sentido, tampoco fue el centro de las reflexiones y análisis alcanzados en los ejercicios investigativos y en los procesos de acompañamiento familiar revisados. Estas perspectivas encontradas representan una concepción pasiva de familia, en la que el logro de objetivos de desarrollo, y con esto, de cambio al interior del grupo, depende más de los recursos y metodologías externas como por ejemplo las instituciones a través del estudiante o el profesional, que de ellas mismas como protagonistas de sus procesos.

En contraste con esta perspectiva de familia como actor pasivo, surge la presente investigación, la cual se inclina por reconocer que el cambio va más allá de la intervención profesional. Esta intervención se constituye en uno de los recursos, no en el único ni en el más importante, dado que, en ocasiones, acudir a la ayuda profesional es la última opción individual o familiar, tras haber intentado una y otra vez resolver la situación, haciendo uso de sus propios recursos y estrategias.

Por lo tanto, la realización de esta investigación se constituye en una oportunidad para que tanto los profesionales que acompañan procesos de trabajo con las familias y sus integrantes, como ellas mismas, reconozcan, valoren y potencien su papel protagónico en los procesos de "desarrollo familiar". Estos procesos pueden entenderse en térmi- 
nos de la consolidación de la calidad de vida de sus integrantes; entendiendo ésta, como un concepto multidimensional. En palabras de Nussbaum (2012), la calidad de vida desde el enfoque de las capacidades implica detenerse en analizar lo que la gente puede "ser o hacer", para Nussbaum "este paradigma parte de una pregunta muy simple: ¿Qué son realmente capaces de hacer y de ser las personas? ¿Y qué oportunidades tienen verdaderamente a su disposición para hacer o ser lo que puedan?" (Nussbaum, 2012, p. 14).

En este mismo sentido, Cebotarev (1979), afirma que la calidad de vida de las personas debe ser entendida como:

Las condiciones necesarias para satisfacer adecuadamente las necesidades básicas y culturalmente definidas requeridas para un "desarrollo normal" del potencial humano de todos los integrantes del grupo familiar y la realización consciente de sus capacidades, sin poner en peligro sus ambientes físico-naturales. (Cebotarev, 1979, p. 16)

De cualquier forma, el mejoramiento de la calidad de vida es una apuesta por activar capacidades para poder funcionar, en este caso, interesa prestar especial atención a las capacidades fortalecidas o desplegadas por las familias para funcionar adecuadamente como familia y enfrentar las situaciones de crisis.

En este sentido, las familias se deben asumir como una realidad social, que es dinámica, compleja y heterogénea, debido a la participación y vinculación de sus integrantes con diversas personas y en diferentes contextos, por la diversidad de estructuras, formas de organización y arreglos familiares que se dan en su interior $y$, por la multiplicidad de relaciones que se construyen. En estos escenarios, se experimentan situaciones de crisis y a su vez procesos permanentes de cambio, generados tanto por las dinámicas internas, como por las interrelaciones entre éstas y el entorno en el que habitan. Las situaciones de crisis familiar hacen que las familias activen sus capacidades (recursos y estrategias) para recuperar o fortalecer sus dinámicas cotidianas, en la mayoría de los casos, sin la ayuda de agentes externos o con una intervención mínima de estos.
En algunas de estas situaciones de crisis, los cambios se relacionan con procesos predecibles, por ejemplo, como el curso de vida de las personas o el ciclo vital de las familias, que sus integrantes pueden ir identificando y realizando, para efectuar los ajustes al interior del grupo mismo, y evitar que se presenten tensiones o conflictos familiares que lleven a pautas de interacción poco favorables a su organización y funcionamiento. Así mismo, se encuentran los cambios ocasionados por la vivencia de situaciones no predecibles, tales como la enfermedad, la muerte, un accidente, el despido laboral de uno de sus integrantes, el secuestro, el desplazamiento forzado, entre otros eventos que generan alteraciones en la dinámica familiar y, por ende, se consideran situaciones de crisis que toman por sorpresa a la familia.

Otras de las situaciones que ocasionan cambios al interior del grupo familiar, tienen que ver con decisiones de carácter personal, que influyen en las relaciones. Es decir, mientras que para algunos es un cambio esperado y provocado, para otros es sorpresivo, ejemplos de esto, pueden ser la separación conyugal, la salida de un hijo del hogar, la conformación inesperada de una relación de pareja, entre otras. En este último aspecto, podría plantearse que algunas de estas decisiones, aunque no dependen del curso de vida ni del ciclo de vida familiar, se relacionan indirectamente con estas etapas.

Las crisis deben considerarse como momentos de la vida cotidiana de las familias y sus integrantes, que ocurren en sus interacciones y pueden en algunas circunstancias conducir a estrategias de cambio con miras a fortalecer las capacidades de las personas para hacerles frente y superarlas exitosamente o, para cualificar ciertas formas de actuación que contribuyen a encontrar formas de relación más democráticas y equitativas tanto dentro como fuera del sistema familiar. Quintero (1997), sostiene que el abordaje sistémico de la familia y el manejo de los conflictos y las crisis familiares, conduce al concepto de "tensión" como un elemento presente en la vida familiar. La autora, se apoya en los planteamientos que hace Frank y Pittman III (1990) (citado en Quintero, 1997) y afirma que:

Una tensión es una fuerza que tiende a distorsionar, es la fuerza específica de cada sistema. 
Puede definirse de acuerdo con varios parámetros. Si es manifiesta u oculta, aislada o habitual, permanente o temporaria, real o imaginaria, universal o específica (...) la tensión no es el factor más importante del problema sino el más inmediato (la última gota) y el punto de acceso más natural y discernible al complejo caos familiar. Para que estalle una crisis debe haber una tensión que exija un cambio y una inflexibilidad que impida que dicho cambio se produzca (Quintero, 1997, p. 98) .

Lo ideal, de acuerdo con Quintero (1997) es que la tensión sea siempre manifiesta, es decir, que pueda definirse claramente porque al hacerlo el camino hacia el cambio tiende a ser mucho más claro y las posibilidades de que la crisis se agudice son menores. Una crisis implica una posibilidad de cambio, de adaptación; razón por la cual, es fundamental, conocer desde las voces de los actores involucrados, los significados atribuidos a ésta, la manera de nombrarla y de ubicarse tanto frente a la situación como frente a las alternativas.

Por su parte, Hernández (1997), sostiene que la familia como sistema, intenta mantener su funcionamiento enfrentando las demandas (estresores y tensiones) con sus capacidades. Esto, debido a que las familias realizan reflexiones y acciones para afrontar las demandas y encontrar las salidas posibles. La demanda mencionada hace referencia a:

Un estímulo o condición que produce o induce un cambio en el sistema familiar, por lo cual puede ser percibida como una amenaza o como un desafío al equilibrio existente. Estas demandas de cambio producen tensión hasta el momento en el que el sistema moviliza alguna capacidad para responder a dicha exigencia; pero cuando no hay una capacidad disponible, aparece un estado de estrés. Así, el estrés no es la presencia en sí misma de un evento o demanda, sino el desequilibrio real o percibido entre demandas y capacidades. (Hernández, 1997, p. 50)

Por su parte, una capacidad es, toda potencialidad que las familias tengan disponibles para afrontar las demandas, según Hernández (1997) leer las capacidades al interior de los grupos familiares es posible a través de dos grandes elementos: los recursos, re- feridos a lo que las familias "tienen", y las estrategias de afrontamiento que dan cuenta de lo que las familias "hacen". Ambos elementos indispensables en la valoración de las posibilidades de cambio que tienen los grupos familiares para resolver exitosamente las diversas situaciones que generan estrés y tensiones en la cotidianidad familiar.

En la fase de adaptación, las familias intentan recuperar y sostener sus dinámicas y relaciones familiares, para lo cual juegan un papel importante los recursos ya disponibles y el reconocimiento de la necesidad de adquirir otros, de acuerdo con las características de la crisis, y los requerimientos propios de cada grupo familiar, para lograr afrontarla exitosamente; es decir, las familias se concentran en la disminución de las demandas y en la resignificación de la situación.

Hernández (1997) afirma que los recursos, pueden ser asumidos como una característica, un rasgo, una competencia o un valor, a nivel individual, familiar o de la comunidad y que éstos pueden ser tangibles (como el dinero) o intangibles (como la autoestima). Identificar estos recursos, se constituye en un ejercicio valioso, debido a que genera claridad acerca de cuáles son los aspectos a los que las familias deben prestarle atención, pues son estos recursos los que, en últimas, garantizan que sea menos traumático para sus integrantes, transitar por diversas etapas de la vida familiar y afrontar los eventos inesperados que se presenten.

El segundo componente de las capacidades son "las estrategias de afrontamiento", entendidas como:

Un esfuerzo específico, abierto o encubierto, por el cual un individuo o una familia como grupo intenta reducir una demanda o exigencia [...] pueden agruparse en patrones, como los que se orientan directamente a mantener la cooperación y la integración familiar; no obstante, cuando el afrontamiento se ubica dentro del contexto de la acumulación de demandas, parece más fácil y relevante verlo como una respuesta generalizada que como una conducta específica. (Hernández, 1997, p. 57)

Si bien, la crisis genera incomodidad y estrés, no puede permitirse que se asuman únicamente como 
características negativas o patológicas de las familias o de alguno de sus integrantes, porque no lo son. Por el contrario, éstas son importantes porque indican que es necesario hacer cambios a nivel individual o familiar, los cuales, por lo general dejan aprendizajes significativos.

Para Fried (2005) la capacidad de afrontamiento alude a:

La recuperación o desarrollo de competencias y recursos para promover conversaciones productivas, coordinaciones sociales, y diseño de los procedimientos necesarios para desplazarse hacia alternativas y nuevas posibilidades. El afrontamiento implica aprendizaje reflexivo, innovación, foco y creatividad social. El diálogo es el medio y el instrumento de este proceso. (2005, p. 3)

Es en este momento, en el que es importante hacer uso de la capacidad de resiliencia de las familias para lograr el cambio, en tanto que, con esta capacidad se busca, afrontar de forma efectiva las dificultades. La resiliencia invita a ubicar el foco de reflexión y análisis en las capacidades y en las alternativas de solución, más que en el problema o en aquello de lo que las familias carecen. Permite que los recursos internos que tienen todos los sistemas humanos (individuos, familias, comunidades, organizaciones, entre otros), sean tenidos siempre en cuenta y activados ante situaciones adversas o críticas de diversa índole. A esto se denomina generar procesos de co-creación y definición de nuevas estrategias en las acciones profesionales y sociofamiliares (Quintero, 2005, p. 5)

Se espera que, dentro de este proceso de cambio permanente surjan nuevos patrones de interacción y con ellos nuevos discursos, que les permitan asumir exitosamente la situación actual de tensión y prepararse para responder a las que emerjan en el futuro. Estos patrones deberían ser el producto de las conversaciones, de los encuentros y desencuentros al interior del grupo familiar.

Estos planteamientos se constituyen en razones para considerar que la familia es un agente de cambio, por cuanto se asume que cada integrante cuenta con capacidades para identificar, reflexionar y co-construir nuevos significados, alrededor de sus propias experiencias de vida. Estas experiencias llevan a la movilización de acciones de cambio a nivel personal y en las relaciones al interior del grupo, lo cual se presenta en esta investigación, en tanto se da cuenta del significado que tuvo para los participantes haber vivido y superado situaciones familiares adversas, lo que se constituyó en una oportunidad para visibilizar su papel protagónico.

\section{Método}

La presente investigación se guio por una metodología cualitativa, entendida como aquella que permite que el investigador se acerque a la experiencia de vida de los sujetos para lograr comprender desde su propia perspectiva, su realidad.

Vasilachis (2006) citando a Marshall y Rossman (1999: 2, 7-8) plantea que este proceso

[...] supone: a) la inmersión en la vida cotidiana de la situación seleccionada para el estudio, b) la valoración y el intento por descubrir la perspectiva de los participantes sobre sus propios mundos, y c) la consideración de la investigación como un proceso interactivo entre el investigador y esos participantes, como descriptiva y analítica y que privilegia las palabras de las personas y su comportamiento observable como datos primarios [...]" (2006, p. 26).

Este tipo de investigación, asume la construcción de conocimiento como un proceso dialógico entre el investigador y los participantes, para esto se realizaron entrevistas a 30 familias urbanas y rurales, residentes en los municipios de Manizales, Neira y Risaralda, en aras de descubrir a través de sus propias voces, el sentido que tuvo haber vivido y superado situaciones familiares adversas, su papel protagónico en estos logros $y$, aportar insumos para cualificar y fortalecer los procesos educativos y de intervención, realizados por instituciones y profesionales de diversas áreas y disciplinas.

El enfoque metodológico fue el hermenéutico, en donde es posible identificar y determinar de manera clara y objetiva lo que se dice y cómo se dice sobre determinada realidad desde un análisis crítico. 
Para la hermenéutica, la principal fuente de información son los protagonistas directos de las experiencias de vida, en tanto que proporcionan información de primera mano. Al respecto, Tójar (2006, p. 122) considera que la hermenéutica "es conocida comúnmente como el arte de interpretar textos con la intención de descubrir su verdadero sentido". Para esto, es preciso anotar que el proceso de interpretación se realizó sobre las narraciones de algunos integrantes de las familias. Una vez transcritas, se procedió a la sistematización de la información, para su codificación y posterior análisis de los datos. Primero, se realizó la "codificación in vivo", en la que el investigador se apoya en los términos usados por los actores participantes, con los que posteriormente se crearon categorías más amplias y se establecieron semejanzas y diferencias. Así, retomando los planteamientos de Glaser y Strauss (citados en Vasilachis, 2006, p. 163) “proceder en un primer paso a maximizar las similitudes y minimizar las diferencias para, luego, en un paso posterior, realizar el proceso inverso, enfatizando las diferencias entre los casos analizados".

En esta investigación participaron 30 familias cuyas experiencias estuvieron representadas en las voces de 34 personas, hombres y mujeres de diversas generaciones y lugares parentales. Es decir, los hombres hablaron como hijos, padres, esposos y abuelos; mientras que las mujeres lo hicieron desde su papel como hijas, madres, esposas, hermanas y tías.

La recolección y selección de la información fue tomada de las conversaciones y diálogos que se tuvieron con las familias a través de la realización de una entrevista semiestructurada, cuyos tópicos fueron la identificación de la situación de crisis familiar, los recursos y estrategias utilizadas por sus integrantes para afrontarla y superarla exitosamente. Luego se procedió a la organización y codificación de los datos; se tomaron de las narraciones, los fragmentos más relevantes para extraer las categorías y subcategorías emergentes, encontrando en éstas aspectos recurrentes y no recurrentes para la interpretación de los datos, etapa en la que se contrastaron las categorías con los datos brindados por los grupos familiares participantes, el referente teórico y la perspectiva de la investigadora.

\section{Resultados y discusión}

\section{Significados construidos alrededor de las crisis: algunos discursos que generan nuevos sentidos}

Las palabras son modos en redes de coordinación de acciones, no representantes abstractos de una realidad independiente de nuestro quehacer. Es por esto que las palabras no son inocuas y no da lo mismo que usemos una u otra en una situación determinada. Las palabras que usamos no solo revelan nuestro pensar, sino que proyectan el curso de nuestro quehacer. (Maturana, 1990, p. 105)

La información que se presenta a continuación parte de la narración realizada por integrantes de las familias alrededor de una situación de crisis vivida, a partir de la cual, se identificaron prácticas y discursos propios de la etapa de afrontamiento del evento y de su posterior superación. Estos dejan ver el significado y el sentido que esta experiencia ha tenido en sus propias vidas y en la cotidianidad familiar. La invitación a la reconstrucción de esta experiencia tuvo como propósito, convertirse en un pretexto para visibilizar el papel protagónico de sus integrantes en el cambio logrado, rescatar y darle valor a los recursos y estrategias implementadas por cada familia para superar exitosamente las situaciones adversas afrontadas $y$, con esto, demostrar que éstas por sus propios medios y con el uso eficiente de sus capacidades, resolvieron crisis normativas y no normativas que ocurrieron, generando aprendizajes y ganancias a nivel personal y familiar.

Responder a este propósito, implica trascender la identificación y descripción de "prácticas" realizadas por las familias, puesto que éstas se explican en función de los significados y sentidos que les otorgan a las experiencias vividas. Es claro que la construcción de significados se logra cuando las personas le otorgan sentido a algo. Al respecto, Lederach como se citó en Ballesteros (2005, 233234), refiere que:

La construcción de significado tiene que ver con el proceso de dar sentido a algo y se logra al relacionar ese algo con otras cosas ya conocidas, 
además el cambio de significado requiere una función de re-encuadre o re-enmarque definido como un proceso mediante el cual algo se reubica y se relaciona con cosas diferentes.

De ahí la importancia de contextualizar al lector, de modo que las palabras de las conversaciones sostenidas con los participantes en el proceso, y que han tenido significado para ellos y ellas, sean comprensibles también para los demás. Conviene subrayar que de lo que las personas hablan cuando se refieren a la vivencia de la crisis, afrontamiento y superación, coincide con lo que se considera significante para ellos, Zlachevsky (2003, p. 48) indica que:

Todos narramos historias [...] la vida de cada uno de nosotros es una historia construida, en donde el actor principal del relato es la persona que nos está relatando los acontecimientos [...] el relato de cada persona es único, y los significados que le atribuyen a los acontecimientos van a depender de la forma particular en que cada persona signifique los acontecimientos que está relatando. Esta forma particular de significar los acontecimientos depende, en gran medida, del sistema u organización de significados que fue adquiriendo a lo largo de la vida, en el convivir con otros, en los espacios de encuentro y desencuentros que tuvo o tiene con otros.

En consonancia con lo anterior, rastrear las prácticas y discursos de las familias, ante la vivencia y superación de algún evento considerado difícil, es importante porque es posible comprender las percepciones que tienen los sujetos frente a sí mismos, frente a los demás y frente a las situaciones y relaciones que estuvieron presentes en este momento de sus vidas. Así como las que dan cuenta del tránsito hacia el cambio buscado por ellos mismos, estos elementos entregan insumos para comprender el significado y el sentido de esta experiencia en sus vidas y sus relaciones familiares.

Los discursos que usan los integrantes de las familias para referirse a las vivencias asociadas a la situación de crisis cambian en el momento en el que se realizan ciertas acciones. Estas acciones generan movimientos en la pauta de interacción y en su correspondiente sentido y significado. Es decir, para que haya un cambio en el discurso, es impres- cindible que cambien los contextos y las relaciones entre las personas involucradas.

Así ocurrió con las familias, durante el proceso de vivir y superar una situación adversa, en la primera etapa de entender la situación, las vivencias que tenían a la mano para relacionar el hecho no fueron las más gratas y alentadoras. Esto debido a que como todo evento estresor generó tensiones que se expresaron en sus prácticas y discursos explícitos, en relación con sus integrantes y en la manera como cada persona se ubicó frente a la situación. Sin embargo, cuando se avanzó en la identificación e implementación de capacidades (recursos y estrategias para afrontar el evento), empezaron a darse cambios positivos. Estos cambios, los llevaron a relacionar nuevamente las experiencias producto de la vivencia de la situación adversa, esta vez, con comportamientos y condiciones mucho más favorables para la familia. De ahí que el significado que estas personas le otorgaron a la crisis familiar, transitó entre la catástrofe y la oportunidad. Entre los significados construidos por las familias alrededor de las experiencias de crisis se destacan los siguientes.

\section{Las crisis como obstáculo en la vida y en las relaciones: Expresiones emocionales asociadas a su vivencia}

“[...] y llega esto (enfermedad del hijo me-
nor) y nos desordena la vida, entonces tam-
bién aprender a vivir con lo que da la vida
¿cierto?, con el caos (...)” (Madrelesposa, en-
trevista personal. Septiembre-Octubre 2014)

Cuando las familias lograron comprender que la situación por la que atravesaron no fue el fin del mundo, y que su opción fue aprender a vivir con lo que la vida trae, como lo expresa esta madre de familia, y que además no fueron las únicas personas que experimentaron una situación similar, dejaron de percibirla como un fracaso. Hernández (1997, p. 65) afirma que por lo general:

La crisis se acompaña de incomodidad y vulnerabilidad, pero no debe tomarse como un factor de estigmatización, calificando la situación co- 
mo un fracaso, o clasificando a la familia como disfuncional e irremediablemente necesitada de ayuda profesional. Por el contrario, hay que comprender que las crisis son situaciones normativas del proceso de desarrollo, que indican el momento de hacer cambios en la estructura y en las reglas familiares.

Pero para que este nivel de comprensión fuera posible, y se diera el cambio, las familias se enfrentaron a una serie de situaciones y emociones, que en ocasiones llegaron a ser favorables a este propósito y en otras se convirtieron en un obstáculo o emergieron nuevas tensiones. Esas últimas hicieron que la definición de la situación no fuera clara y, con esto que el trascurso por la situación de conflicto fuera más complejo y difícil de afrontar; así lo refiere Hernández (1997):

Las familias atraviesan repetidos ciclos de ajuste-crisis-adaptación, desencadenados algunos por los eventos propios del desarrollo (nacimiento del primer hijo, ingreso a la escuela, etc.), los cuales producen crisis normativas o transiciones que son seguidas por períodos de relativa estabilidad. Otros ciclos se inician por la aparición de un estresor no normativo, como el diagnóstico de una enfermedad severa en algunos de los miembros, ante lo cual se produce una crisis, dado que las demandas exceden las capacidades de las familias, al menos transitoriamente. Viene luego la fase de adaptación durante la cual se restaura el equilibrio y se instaura la estabilidad relativa hasta la siguiente crisis. (1997, p. 62)

Una de las tendencias que se identificaron en los relatos de las personas, dio cuenta de que hay quienes consideraron que las crisis experimentadas por ellos y sus familias fueron asumidas como situaciones que "desestabilizaron" y "conmocionaron sus vidas", en tanto fue un hecho difícil de entender y manejar. Esto se dio durante el tránsito por la situación de crisis, y en algunos casos, luego de haberla superado, o de al menos comprenderla y asimilarla.

Los discursos con los cuales asociaron inicialmente la situación experimentada al interior de sus familias fueron: "catástrofe", "adversidad" y "caos" a raíz del impacto a nivel individual y familiar que ésta generó. Es válido señalar que un discurso es mucho más que unas palabras, porque están cargados de emociones y prácticas cotidianas, las cuales se derivan de los significados que cada persona construye a lo largo de la vida,

Restrepo (1999), citando a Fowler (1985) y Spender (1984), indica que: "La gente usa el lenguaje para comunicar sus pensamientos, ideas, creencias y sentimientos a otros y, al mismo tiempo, obtener algo de ellos por medio de una respuesta. Es una práctica social de construcción de la realidad" (1999, p. 69). De ahí la necesidad de referirse a lo que ocurrió en las dinámicas y relaciones familiares, dado que esto es lo que lleva a las personas a asociar la experiencia con cierto tipo de discursos y no con otros.

Los entrevistados recordaron que en su afán por resolver la situación; es decir, la fase de ajuste, experimentaron otra serie de circunstancias difíciles como el hecho de tener que irse a otra ciudad y alejarse de su red de apoyo familiar. La pérdida de figuras de autoridad, de protección y de afecto, asumir responsabilidades económicas y de cuidado ante la muerte de quienes antes se encargaban de cumplir con estas tareas (padre o esposo, madre, hermanas); la desaparición -o disminución- de espacios de encuentro familiar y, el darse cuenta de que un integrante de la familia (hijos o padres) presentaba una enfermedad incurable; fueron algunas de las explicaciones que dieron cuenta de las razones por las cuales esa vivencia inicialmente fue asociada a discursos que giraron en torno al "caos" y la "tragedia".

Esta particular manera de referirse a las crisis tuvo que ver con todo lo que estaba ocurriendo a su alrededor, leído a través de las acciones realizadas por algunos de los integrantes de las familias y los efectos de éstas en su cotidianidad y en las relaciones. En este orden de ideas, Fried (2005, p. 2) señala que:

Cuando las personas se encuentran en situaciones de crisis y/o conflictos con frecuencia experimentan desorientación y turbulencias afectivas, y responden con desencuentros serios, intentos parciales o inestables de reiterar soluciones exitosas en el pasado, pero no ade- 
cuadas en el presente. Son instancias que movilizan fuertes emociones, de desafío, estrés, estupor, dolor, desconcierto en quienes están involucrados y necesitan poder manejarlas en búsqueda de alternativas viables.

Pero mientras esto ocurrió fue inevitable que sus protagonistas experimentaran una serie de emociones, las cuales durante el proceso de afrontamiento no podían desconocerse, porque en éstas existían posibilidades para la construcción de nuevos significados y sentidos, útiles en la visibilización de caminos posibles para la superación de la crisis, el reconocimiento y valoración de las capacidades individuales y familiares.

Teniendo en cuenta la importancia de la comprensión del papel de las emociones en las interacciones cotidianas y su relación con el cambio, se precisa exponer algunas de las ideas de Maturana (1990), quien sostiene que lo que caracteriza a lo humano no es propiamente su capacidad de razonar, sino la interrelación entre la razón y la emoción. Cuando se habla de las emociones, la dificultad percibida por el autor tiene que ver con que estas emociones comúnmente carecen de reconocimiento y se asumen como opuestas a la razón; es decir, no se tiene en cuenta que, para comprender las relaciones humanas, es preciso entender las emociones, dado que son éstas las que las sustentan y definen.

Cuando uno cambia de emoción, cambia de dominio de acción. En verdad, todos sabemos esto en la praxis de la vida cotidiana, pero lo negamos, porque insistimos en que lo que define nuestras conductas como humanos es su ser racional. Al mismo tiempo todos sabemos que cuando estamos en una cierta emoción hay cosas que podemos hacer y cosas que no podemos hacer, y que aceptamos como válidos ciertos argumentos que no aceptaríamos bajo otra emoción (1990, p. 15-16).

Tal y como lo muestran los hallazgos de la investigación, en donde una situación de crisis familiar llevó al surgimiento de múltiples emociones que acompañaron los sujetos en su individualidad. Esto fue útil para entender, tanto las percepciones frente a sí mismo y frente al episodio experimentado, como las características de las interacciones entre éste y las personas familiares que directa e indirectamente tenían que ver con la situación. La emoción es un componente de análisis central a la hora de leer y comprender los comportamientos humanos, puesto que éstas se generan, construyen y mantienen en la interacción con otros.

Entre las expresiones emocionales presentadas de manera reiterada en los relatos de las personas entrevistadas $y$, que dieron cuenta de sus opiniones frente a la vivencia de la crisis familiar, se destacaron la tristeza por el dolor propio y el de los demás miembros de sus familias, al presenciar el sufrimiento de los hijos o el maltrato experimentado por la madre en su relación de pareja, por haber estado enfrentada a señalamientos al no responder al perfil de mujer, madre o hija que la cultura ha idealizado y reforzado, por la aparición de enfermedades de difícil tratamiento, incurables o discapacidades que llevaron a algún integrante de la familia a ubicarse en un estado de fragilidad o vulnerabilidad; la angustia ante el debilitamiento de las relaciones familiares, y la vergüenza al no cumplir con las expectativas sociales y las de la misma familia.

En efecto, en estas familias se reportó una sensación de contradicción y confusión al momento de tomar decisiones, la impotencia porque la situación escapó del control, la rabia y la desesperación al no encontrar respaldo en algunos de los integrantes de su familia (padre, madre o esposo) considerados importantes en su vida. Así mismo, se reportó la experimentación del sentimiento de culpa y resignación ante lo que para ellos en ese momento no tenía salida. Esto hizo que en algunos casos un integrante de la familia presentara episodios de depresión y que se generaran conflictos en las relaciones, viéndose debilitados los vínculos familiares y poniendo en riesgo la unión familiar, considerada como un recurso importante en el afrontamiento y superación de las situaciones de crisis.

Así mismo, existió temor ante lo desconocido, ante la incertidumbre de no conocer el rumbo que tomaría la situación y sobretodo el no saber si la familia o alguno de sus integrantes disponían, o podrían adquirir, las herramientas para hacerle frente a la situación y superarla. Las historias narradas mostraron que les fue difícil entender lo que estaba 
ocurriendo con sus familias, en ocasiones sentían que éstas "se estaban desbaratando", y les atormentaba ser testigos del dolor y el sufrimiento de algunos de sus seres queridos y no poder hacer nada para evitarlo.

Por lo general, lo que ocurrió fue que se acentuaron otras crisis o tensiones no resueltas o aparecieron nuevas tensiones dada la dificultad para coordinarse, llevando a la aparición de relaciones tensas y con esto, al distanciamiento o a prácticas cotidianas violentas, que poco ayudaron a la familia a que afrontaran la situación de crisis. Al respecto, Fried (2005, p. 7) indica que en estos casos:

Progresivamente se incrementan la inculpación recíproca, el enojo, el resentimiento, la intolerancia, y las discusiones aparecen y desaparecen de acuerdo a patrones azarosos. La desorganización de los patrones y creencias, y la pérdida del consenso llevan a que cada miembro sienta que no sabe quién es -ni como persona ni dentro de la organización- y hasta sienta que su vida se disuelve, que sus vínculos con otros y su pasado se tornan vulnerables y pueden romperse.

Cabe señalar, que para algunos de los entrevistados vivir esto fue una experiencia dolorosa porque adicional a esto, hubo momentos de "soledad", a raíz del distanciamiento familiar, expresado en el hecho de que "cada quien cogió su camino". Algunos voluntariamente porque deseaban alejarse y evadir conversaciones relacionadas con la situación, o porque los adultos, en su mayoría mujeres y madres, se vieron obligadas a trabajar largos periodos de tiempo o a irse a otros lugares en busca de empleo para satisfacer las necesidades de sus hijos, y el costo de esto, fue tener que dejarlos solos, sin seguimiento ni control frente a sus comportamientos, porque las redes de apoyo no eran visibles, o no estaban a su disposición en ese momento.

Este panorama, inicialmente desalentador para quienes enfrentaron cualquier tipo de situación adversa, llevó a que algunos de sus integrantes encontraran en el intento de suicidio ( 3 familias) y en el alcohol ( 2 familias) una salida posible, porque no fue fácil para ellos comprender la situación y creer que ésta podría resolverse por otra vía, haciendo más difícil el manejo del evento familiar.

Por su parte, cuando se menciona qué factores o actores externos a la familia, habían desencadenado la vivencia de la situación de crisis, aparecieron el odio y la rabia como emociones que llevaron a pensar en la venganza. Esto ocurrió en los casos en que alguien le quitó la vida a un integrante de su familia o causó daño físico o psicológico. Estas se constituyeron en expresiones emocionales que se mezclaron a su vez con el miedo y la desconfianza en sí mismo y en los otros.

Al respecto, Fried (2005) asegura que las dificultades en el afrontamiento exitoso de las situaciones de crisis se dan cuando:

Disminuyen el consenso y la habilidad para actuar coordinadamente, se opaca el sentido de identidad y las expectativas recíprocas, se cuestiona la validez de las relaciones; se genera aislamiento; se genera inculpación recíproca; se incrementan la rigidez y las demandas de un funcionamiento que ya no es necesariamente válido, las personas pierden la capacidad de tomar responsabilidad; se disuelve la trama social y a veces se llega a una crisis profunda o a una ruptura. (2005; p. 4-5) .

Las dificultades de afrontamiento, experimentadas por los integrantes de las familias, estuvieron directamente asociadas con lo que plantea Fried (2005). Es así como, en algunas de las historias, afloró además una sensación de humillación, por varias razones. Una de estas, porque las personas que al parecer estaban apoyando a las familias, con sus actos hicieron que éstos se sintieran inferiores, más vulnerables $y$, porque algunas de las mujeres y esposas consideraron que al intentar mantener su relación de pareja, realizaron acciones que iban en contra de su dignidad como persona, de su amor propio, y de las cuales hoy una vez comprendida y superada la situación, se arrepienten. Finalmente la "desconfianza", generada tras los engaños y decepciones experimentadas en las relaciones $y$, porque las personas asumieron que no necesitaban ayuda de nadie para afrontar la situación, en tanto sintieron que contaban con la suficiente fuerza para hacerlo solos, o porque pensaron que 
en ese momento, nadie podía entender lo que les estaba pasando.

Hasta este punto, se ha dado cuenta de las expresiones emocionales identificadas en algunas narraciones de los actores consultados, los cuales permiten concluir que ésta fue en su inicio, una experiencia difícil para las familias, con lo que se reitera la necesidad de hacerlas visibles, de modo que sea posible para ellas mismas o con la ayuda de un agente externo, trabajarlas, hacer que se generen nuevos significados y sentidos útiles para el cambio requerido y anhelado por cada grupo familiar; cambios que de acuerdo con Sánchez (2016, p. 95), se hacen evidentes:

En el momento en que se logran otros significados o variaciones sobre significados que se originan en la conversación. El cambio es inherente al diálogo y se da en el contar y recontar las historias familiares, en las redefiniciones y en los diferentes significados conferidos a los sucesos del pasado, del presente y del futuro imaginado.

Es claro hasta aquí, que los episodios ocurridos durante la vivencia de la crisis familiar, les permitieron a sus integrantes, la co-construcción de nuevos significados y sentidos alrededor de las prácticas realizadas para hacerle frente a la situación. Es decir, pasar de discursos negativos, a hacer énfasis en la esperanza, la solidaridad, la unión, la fortaleza e incluso la resignación, lo que es posible sólo cuando se transforma la emoción.

\section{Conclusiones}

A partir de este trabajo se concluye que se requiere visibilizar a través de las prácticas y los discursos las emociones positivas y negativas en las que se soportan las dinámicas y relaciones cotidianas, para develar en éstas los nuevos sentidos y significados otorgados por los integrantes de las familias a la situación de crisis. Esta visibilización, se constituye en una tarea importante para lograr el cambio, puesto que en la medida en que se hagan conscientes "las palabras" y señales del lenguaje, será posible comprender lo qué las personas sienten y piensan, y de este modo, orientar acciones hacia la resignificación de esas emociones, como fundamentos que constituyen sus acciones.

Partir de las propias experiencias y capacidades, se constituye en un aporte decisivo, digno de seguirse cualificando. De modo tal que cada vez más los cursos de acción de las personas que conforman los grupos familiares, de las instituciones que en ocasiones operan como redes de apoyo, de los agentes educativos y los profesionales, estén centrados en prácticas relacionales y señales del lenguaje generativos, que hagan posible ver en el otro, un sujeto con potencialidades y fortalezas, más que con problemas y dificultades, puesto que cuando esto no ocurre, las acciones-prácticas relacionales asociadas dan cuenta de la dificultad para la coordinación, porque las acciones están concentradas en la confrontación, en la culpabilización y en la invisibilización del otro, no hay consenso y hay dificultades en la toma de decisiones acertadas, lo que aumenta la sensación de no pertenencia al grupo familiar.

Pero si se reconoce que el ser humano como ser social se construye cotidianamente, y que el diálogo es un mecanismo apropiado para hacer que cambien los significados que las personas elaboran alrededor de "alguien" o de "algo", las emociones pueden ser reorientadas positivamente. Es así como se logra identificar en las conversaciones sostenidas con las familias, que hay un momento en la etapa de afrontamiento de su experiencia adversa, en el que empiezan a aparecer como expresiones emocionales centrales la solidaridad, la fe, la unión e incluso como ellos mismos lo manifiestan la resignación; las cuales inmediatamente modifican los cursos de acción al interior de la familia y con esto, las relaciones en las que empiezan a ser visibles la emergencia de espacios abiertos para la comunicación, la disposición de sus integrantes para encontrar salidas a favor del colectivo, el entendimiento y la cohesión familiar. Prácticas y discursos generativos que les permitieron encontrar nuevas posibilidades, las cuales enriquecieron su vida personal y familiar.

Tanto a los integrantes de las familias como a los profesionales, que en algunos casos deben intervenir en el acompañamiento, les compete hacer que las conversaciones sostenidas alrededor de una 
situación familiar, se constituyan en un pretexto conducente a reforzar o visibilizar en los casos en que sea necesario, la idea del sujeto como agente protagónico de sus propios procesos de cambio, y de lo que significa en su búsqueda, hacer uso o potencializar los recursos individuales, familiares, comunitarios y las estrategias de afrontamiento.

\section{Referencias}

Ballesteros, B. (2005). El concepto de significado desde el análisis del comportamiento y otras perspectivas. Universitas Psychologica, 4(2), 231-244.

Cebotarev, N. (1979). Mujer Rural y Desarrollo: Nuevo enfoque de la Educación del hogar en América Latina. Bogotá: Centro Internacional de Investigaciones para el Desarrollo (IDRC/CIID).

Fried, D. (2005). Afrontamiento de crisis y conflictos: una perspectiva generativa. Recuperado de http://fundacioninterfas.org/capacitacion/ wp-content/uploads/2013/10/7AfrontGenerati voCrisisConflictosOrganizaciones.pdf.

Hernández, Á. (1997). "Familia ciclo vital y psicoterapia sistémica breve. Santafé de Bogotá: Editorial Códice.

Maturana, H. (1990). Emociones y lenguaje en educación y política. Centro de estudios del desarrollo -CED-. Dolmen Ediciones S.A.
Nussbaum, M. (2012). Crear capacidades. Propuesta para el desarrollo humano. Barcelona: editorial Paidós.

Quintero, Á. (1997). Trabajo social y procesos familiares. Buenos Aires: Lumen/Humanitas.

Quintero Á. (2005). Resiliencia: Contexto no clínico para trabajo social. Revista Latinoamericana de Ciencias Sociales, Niñez y Juventud, 3(1), 1-16.

Restrepo. D. (1999). Desigualdad de género. Privilegios y derechos culturales en familias de Caldas. Bogotá: Instituto Colombiano para el Fomento de la Educación Superior.

Sánchez, M. (2016). Movimientos sociolingüísticos en las conversaciones terapéuticas. Hacia los lenguajes del cambio. Manizales: Editorial Universidad de Caldas.

Tójar, J. (2006). Investigación cualitativa, comprender y actuar. Madrid: Editorial la Muralla S.A.

Vasilachis, I., Ameigeiras, Aldo., Chernobilsky, L., Giménez. V., ... Soneira., A. (2006). La investigación cualitativa. Estrategias de investigación cualitativa. Barcelona: Editorial Gedisa.

Zlachevsky O. A. M. (2003). Psicoterapia Sistémica Centrada En Narrativas: Una Aproximación. Revista Límite, 10, 47-64. 